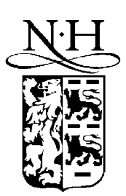

ELSEVIER

\title{
Evaluation of a force sensor based on a quartz tuning fork for operation at low temperatures and ultrahigh vacuum
}

\author{
S. Hembacher*, F.J. Giessibl, J. Mannhart \\ Experimentalphysik VI, Center for Electronic Correlations and Magnetism, Augsburg University, D-86135 Augsburg, Germany
}

Received 2 September 2001; accepted 1 October 2001

\begin{abstract}
The noise performance of the force sensor is crucial for optimizing the resolution in non-contact atomic force microscopy. Sensing forces in vacuum and low temperatures is even more demanding than at ambient conditions. Here we analyze the noise performance and the sensitivity of a force sensor based on a quartz tuning fork of which one of the prongs is fixed (qPlus sensor). The noise characteristic of the qPlus sensor, optical and piezoresistive detection schemes at room temperature are compared and the qPlus sensor is investigated at low temperatures. The frequency variation of quartz tuning forks as a function of temperature is experimentally determined for the temperature range from 4 to $300 \mathrm{~K}$. (C) 2002 Elsevier Science B.V. All rights reserved.
\end{abstract}

Keywords: Non-contact AFM; Low temperatures; qPlus sensor

\section{Introduction}

Since Binnig et al. [1] introduced the atomic force microscope (AFM) in 1986, many different operation modes have been developed for this instrument. From a pure static contact mode to the dynamic non-contact mode with large amplitudes which reaches true atomic resolution on reactive surfaces like the $\operatorname{Si}\left(\begin{array}{lll}1 & 1 & 1\end{array}\right)\left(\begin{array}{l}7 \\ \times\end{array}\right)$ [2], the resolution has been steadily increased. The noise characteristics and the sensitivity of an atomic force sensor are responsible for the quality of the measurements in non-contact atomic force microscopy. Typically, a force sensor for AFM consists of a cantilever and a deflection measurement scheme. The experimental deflection noise is determined by

\footnotetext{
* Corresponding author. Tel.: +49-821-598-3662; fax: +49-821-598-3652.

E-mail address: stefan.hembacher@physik.uni-augsburg.de (S. Hembacher).
}

both the cantilever and the deflection measurement scheme.

In principle, two different kinds of detection systems can be distinguished:

- self-sensing devices such as piezoresistive [3] or piezoelectric cantilevers [4];

- cantilevers with an external deflection measurement scheme such as a tunneling tip [1], the beam deflection technique $[5,6]$ or the interferometric technique [7].

While operation in vacuum and low temperatures has been demonstrated with the tunneling [8] and the interferometric techniques [9-12], cantilevers with self-sensing capability are particularly desirable for operation in vacuum and low temperature because of the great difficulties involved with the alignment of a tunneling tip, optical fiber or laser beam in these environments. Low-temperature operation of piezoresistive cantilevers has been demonstrated by Volodin 
and Van Haesendonk [13], and piezoelectric sensors have been operated at low temperature by Rychen et al. [14].

Excellent resolution at room temperature has already been demonstrated with the qPlus sensor [15] using optimized imaging parameters [16]. Because thermal drift is strongly reduced and the bandwidth can be reduced greatly, even better resolution is expected at low temperatures. In addition to working with stiff cantilevers, it is important to use small amplitudes in the Angström range to achieve this resolution [17]. To realize such small amplitudes in a controlled manner, the noise of the force detection unit must be as small as possible. The spectral deflection noise density is a quantity for noise calibration and expressed by the ratio between the deflection noise and the bandwidth.

\section{Measurement at room temperature}

As described, the deflection of a cantilever can be measured by optical, piezoresistive and piezoelectric means. Ultimately, all systems convert the cantilever deflection into a voltage. For the qPlus sensor, Fig. 1 shows the sensor and its measurement circuit necessary to convert the deflection into an output voltage. For the measurements presented here, the operational amplifier AD711 [18] and a $60 \mathrm{M} \Omega$ feedback resistor is used. Because the amplification is proportional to $R$ and the Johnson noise of the resistor is proportional to $R^{1 / 2}$ an optimal signal-to-noise ratio is expected for infinitely large values of $R$. However, the parasitic capacitance of the resistor reduces the bandwidth of the amplifier. Therefore an optimal value exists for the feedback resistor which is usually found empirically.

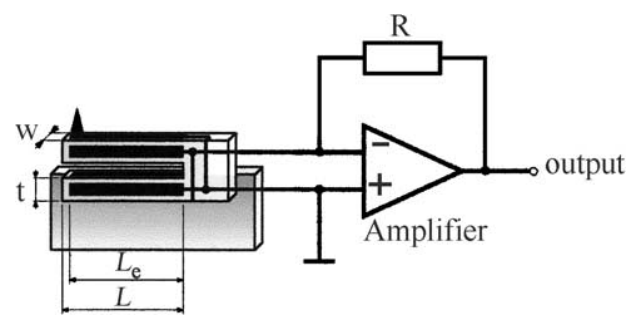

Fig. 1. Measurement circuit for a qPlus sensor. The preamplifier is a current-to-voltage converter with a high bandwidth similar to a preamplifier in a scanning tunneling microscope.

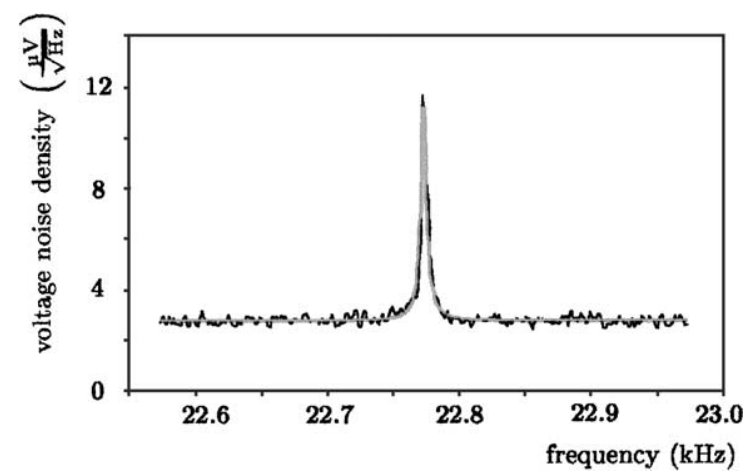

Fig. 2. Spectral voltage noise density at the output of a current-tovoltage converter connected to a qPlus sensor at room temperature.

We define the sensitivity of a force sensor by the delivered voltage generated by a full deflection. To determine this value, the output voltage of a deflection system of a force sensor due to the thermal activation of the cantilever is determined. A typical frequency spectra is shown in Fig. 2.

A fit of the spectral voltage density $V(f)$ data with a free-vibration model

$V(f)=\sqrt{U_{\text {noise }}^{2}+\frac{V_{\text {sensor }}^{2} f_{0}^{4}}{\left(f_{0}^{2}-f^{2}\right)^{2}+\left(f_{0} f / Q\right)^{2}}}$

yields the spectral white noise voltage density $V_{\text {noise, }}$ the eigenfrequency $f_{0}$ and the quality factor $Q$. An integration over the measured bandwidth and the subtraction of the noise floor yields the voltage $V_{\text {therm }}$ of the sensor due to the thermal activation. To obtain the sensitivity of the sensor, the thermal-activated vibration amplitude has to be determined. At a certain temperature $T$, and if thermal equilibrium is reached, the amplitude $\left\langle z_{\text {therm }}\right\rangle$ equals $\sqrt{k_{\mathrm{B}} T / k}$ for a cantilever with stiffness $k$. Here $k_{\mathrm{B}}$ is the Boltzmann constant. The ratio

$S(T, k, f)=\frac{V_{\text {therm }}}{\left\langle z_{\text {therm }}\right\rangle}$

is the sensitivity of the combination of the sensor plus its deflection system. For a qPlus sensor the sensitivity is given by [4]

$S(f)=3 d_{12} E_{\mathrm{Y}} w \frac{L_{\mathrm{e}} t}{L^{3}}\left(\frac{L_{\mathrm{e}}}{2}-L\right) 2 \pi f R$ 
Table 1

Comparison of sensitivity and noise level of a micro-machined silicon cantilever with beam deflection detection, a piezoresistive cantilever and the qPlus sensor

\begin{tabular}{lcccc}
\hline & $k(\mathrm{~N} / \mathrm{m})$ & $f_{0}(\mathrm{~Hz})$ & $S(\mu \mathrm{V} / \mathrm{pm})$ & $\tilde{z}(\mathrm{fm} / \sqrt{\mathrm{Hz}})$ \\
\hline Beam deflection detection & 2 & 66192 & 1.6 & 2400 \\
Piezoresistive cantilever & 6.3 & 83050 & 0.57 & 1300 \\
qPlus sensor & 1800 & 28773 & 17.5 & 159 \\
\hline
\end{tabular}

where $E_{\mathrm{Y}}$ is the Young's modulus, $L$ the length and $t$ the thickness of the prongs, $L_{\mathrm{e}}$ the length of the electrodes of the tuning fork and $d_{12}=2.1 \mathrm{pC} / \mathrm{m}$ [19] the piezoelectric coupling constant. $R$ is the value of the feedback resistor (see Fig. 1) provided that the operational amplifier is ideal such that the gain of the current-to-voltage converter is given by $R$. It can be seen from Eq. (3) that the sensitivity of a qPlus sensor is a linear function of the eigenfrequency of the sensor.

The relevant value for the comparison of different force sensors is the spectral amplitude noise density which is given by the ratio of the white noise level and the sensitivity of the sensor including its deflection system

$\tilde{z}=\frac{U_{\text {noise }}}{S}$

The spectral noise density determines the resolution which is achievable by the AFM [20]. As outlined before, the smaller the vibration amplitude of the cantilever, the more sensitive the measurement is on short range forces, i.e. chemical forces.

Table 1 displays the results measured for room temperature operation of a micro-machined silicon cantilever with beam deflection detection, a piezoresistive cantilever and the qPlus sensor.

The measurements with the standard silicon cantilever detected with a laser beam are performed under ambient conditions. For a fiber optic interferometer, where the cantilever is replaced by a polished silicon wafer, a deflection noise density of $50 \mathrm{fm} / \sqrt{(\mathrm{Hz})}$ has been quoted. However, the deflection noise density for a real cantilever was between 0.1 and $10 \mathrm{pm} / \sqrt{(\mathrm{Hz})}$ [21]. The measurements on the piezoresistive cantilever and the qPlus sensor is competitive with the noise of optical deflection measurement systems. By using Eq. (3) with $R=60 \mathrm{M} \Omega$ and $d_{12}=2.1 \mathrm{pC} / \mathrm{m}$ [19], the value for the theoretical sensitivity is $21.9 \mu \mathrm{V} / \mathrm{pm}$, which fits well to the measured data.
In Ref. [4], the experimental sensitivity was reported to be only half of the theoretical value. In the process of the experiments presented here, we found that this deviation was due to an insufficient bandwidth of the preamplifier used in Ref. [4].

\section{Measurement at low temperatures}

At low temperatures, we expect a reduced thermal noise because the dominant contribution to the white noise is due to the Johnson noise of the feedback resistor of the current-to-voltage converter. In addition, Eq. (3) predicts an increased sensitivity because $d_{12}$ rises about $10 \%$ and the Young's modulus rises about $1 \%$ if the temperature decreases from 300 to $5 \mathrm{~K}$ [19]. Both the effects lead to a decreasing amplitude noise density of the qPlus sensor.

First of all we have determined the temperaturedependent relative frequency change of our used tuning fork ${ }^{1}$ to estimate the temperature of the qPlus sensor. These measurements are done in a flow cryostat using an encapsulated tuning fork which is excited externally. The results of these measurements are shown in Fig. 3.

First the measurements have shown that the relative frequency change of the qPlus sensor is much larger than the frequency change of a tuning fork in its metal case for a temperature range from 300 to $77 \mathrm{~K}$. For the temperature range from 77 to $4 \mathrm{~K}$, the relative frequency changes are similar for the qPlus sensors and the encapsuled tuning forks. For minimizing the white noise density of the force detector output, the capacitance of the leads connecting the qPlus sensor to the current-to-voltage converter has to be minimized. Because this capacitance increases with the length

\footnotetext{
${ }^{1}$ We have used the tuning fork type DS26 manufactured by Micro Crystal [22].
} 


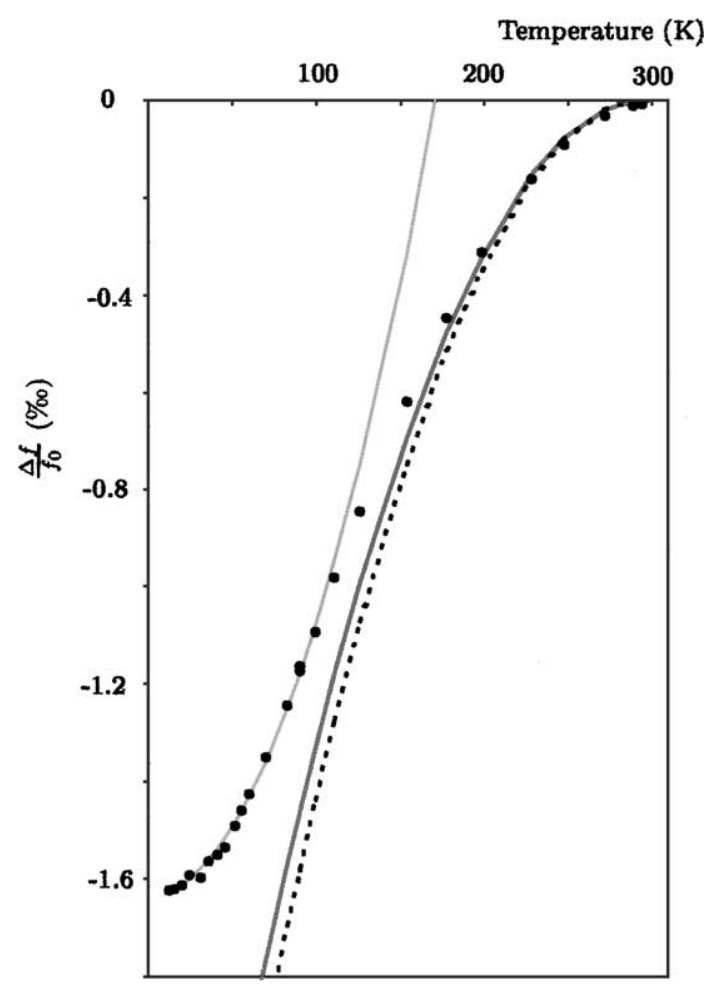

Fig. 3. Relative frequency change as a function of temperature for a quartz tuning fork. The filled circles in the diagram represent the measured data. The dotted line is the fitting curve of the manufacturer. The solid lines are the fitting curves for the temperature range between $300-200 \mathrm{~K}$ and $0-100 \mathrm{~K}$.

of the leads, the preamplifier must be positioned close to the qPlus sensor. The operational amplifier that we use works properly only at temperatures above $-40{ }^{\circ} \mathrm{C}$. Therefore we equipped the amplifier with a heater resistor and enclosed this assembly in a metal case for thermal shielding. The qPlus sensor is hereby mounted directly on a copper cylinder which has direct contact to the cooling liquid. The temperature measurement is done with a Si-diode also mounted on the copper block near the sensor too. With this arrangement, it is possible to determine the thermal vibration amplitude and measure the sensitivity and the spectral amplitude noise density as a function of temperature. During the measurements, the copper block is pulled out of the liquid nitrogen or helium because the vibrations caused by the boiling liquids cause mechanical excitation in addition to the thermal excitation of the qPlus sensor.
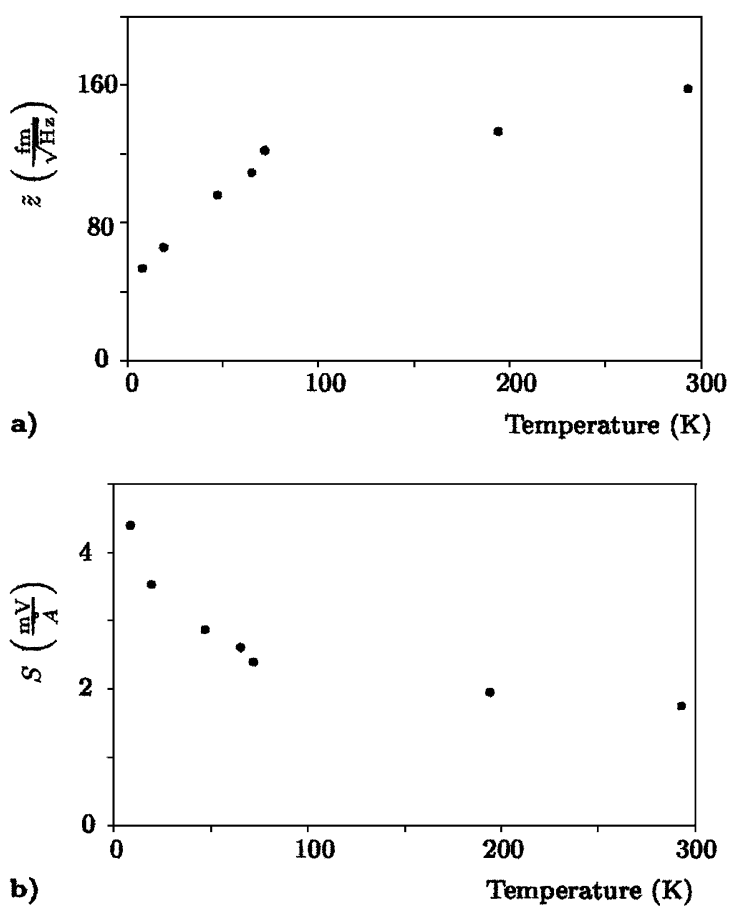

Fig. 4. (a) Amplitude noise density $\tilde{z}$ and (b) sensitivity $S$ as a function of temperature.

The results for the temperature-dependent amplitude noise density and the sensitivity are summarized in Fig. 4. As expected, the deflection noise density $\tilde{z}$ decreases with temperature. While an increase of the sensitivity for decreasing temperature is expected from the increase of $d_{12}$ with decreasing temperature, the observed increase significantly exceeds the anticipated one. Possibly even though we have removed the copper block from the boiling liquid, some mechanical excitation remain. To resolve this issue, we therefore plan measurements in which the qPlus sensor is deliberately excited mechanically.

\section{Summary and conclusion}

We have shown that the qPlus sensor functions at low temperatures with an even better noise performance than at room temperature. Because the spectral deflection noise density decreases with temperature, an AFM equipped with the qPlus sensor is expected to achieve at low temperatures an even better resolution than at $300 \mathrm{~K}$. 
The variation of eigenfrequency as a function of temperature has been determined experimentally for a temperature range from 4 to $300 \mathrm{~K}$. As shown in Fig. 3, the temperature induced relative frequency change almost vanishing both at 4.2 and $300 \mathrm{~K}$. Therefore at these temperatures, the influence of a temperature variation to the eigenfrequency of the cantilever is minimized. The results underline the capability of the qPlus sensor as an extremely sensitive force sensor even at low temperatures.

\section{Acknowledgements}

This work is supported by the BMBF (Project No. 13N6918).

\section{References}

[1] G. Binnig, C.F. Quate, Ch. Gerber, Phys. Rev. Lett. 56 (1986) 930.

[2] F.J. Giessibl, Science 267 (1995) 68.

[3] M. Tortonese, R.C. Barrett, C.F. Quate, Appl. Phys. Lett. 62 (1993) 843.

[4] F.J. Giessibl, Appl. Phys. Lett. 76 (2000) 1470.

[5] G. Meyer, M. Amer, Appl. Phys. Lett. 53 (1988) 1045.
[6] S. Alexander, L. Hellemans, O. Marti, V. Elings, P.K. Hansma, M. Longmire, J. Gurley, J. Appl. Phys. 65 (1) (1989) 164.

[7] G.M. McClelland, R. Erlandson, S. Chiang, in: D.O. Thompson, D.E. Chimenti (Eds.), Review of Progress in Quantitative Nondestructive Evaluation, Vol. 6, 1987.

[8] F.J. Giessibl, Ch. Gerber, G. Binnig, J. Vac. Sci. Technol. B 9 (1991) 984.

[9] H.J. Hug, Ph.D. Thesis, University of Basel, Switzerland, 1993.

[10] W. Allers, A. Schwarz, U.D. Schwarz, R. Wiesendanger, Rev. Sci. Instrum. 69 (1) (1998) 221.

[11] H.J. Hug, B. Stiefel, P.J.A. Schendel, A. Moser, S. Martin, H.J. Güntherodt, Rev. Sci. Instrum. 70 (9) (1999) 3625.

[12] M. Roseman, P. Gruetter, Rev. Sci. Instrum. 71 (10) (2000) 3782.

[13] A. Volodin, C. Van Haesendonk, Appl. Phys. A 66 (1998) S305.

[14] J. Rychen, T. Ihn, P. Studerus, A. Herrmann, K. Ensslin, Rev. Sci. Instrum. 70 (1999) 2765.

[15] F.J. Giessibl, S. Hembacher, H. Bielefeldt, J. Mannhart, Science 289 (2000) 422.

[16] F.J. Giessibl, H. Bielefeldt, S. Hembacher, J. Mannhart, Appl. Surf. Sci. 140 (1999) 352.

[17] F.J. Giessibl, H. Bielefeldt, S. Hembacher, J. Mannhart, Ann. Phys. 10 (2001).

[18] Analog Devices. http://www.analog.com.

[19] A.G. Beda, Sov. Phys. 9 (1997) 1043.

[20] F.J. Giessibl, Habilitation Thesis, Augsburg University, 2000.

[21] D. Rugar, H.J. Marmin, P. Guethner, Appl. Phys. Lett. 55 (1989) 2588.

[22] Micro Crystal, Switzerland, http://www.mcrystal.ch. 